\title{
Desempenho, medidas corporais, rendimentos de carcaça e cortes, e qualidade de carne em cordeiros alimentados com resíduos da agroindústria processadora de frutas
}

\section{Performance, body measurements, carcass and cut yields, and meat quality in lambs fed residues from processing agroindustry of fruits}

\author{
Júlio César Silva Almeida ${ }^{1}$; Darcilene Maria de Figueiredo ${ }^{2 *}$; \\ Cleube Andrade Boari ${ }^{1}$; Mônica Lopes Paixão ${ }^{3}$; Janaina Adna Barbosa Sena²; \\ Janaina Leite Barbosa ${ }^{2}$; Marluci Olicio Ortênción ${ }^{4}$ Kariny Ferreira Moreira ${ }^{5}$
}

\section{Resumo}

\begin{abstract}
Esta pesquisa foi conduzida com o objetivo de se avaliar a utilização dos resíduos de abacaxi (Ananas comosus L.), banana (Musa sp.), manga (Mangifera indica) e maracujá (Passiflora ssp.) na alimentação de cordeiros confinados, sobre desempenho produtivo, rendimento de carcaça e características qualitativas e quantitativas da carne confinados. Foram utilizados vinte e cinco ovinos mestiços com a Raça Santa Inês e sem raça definida, distribuída em cinco tratamentos. Os tratamentos foram constituídos pela substituição de $75 \%$ da silagem de sorgo pelos respectivos coprodutos, sendo que no tratamento controle o volumoso foi composto apenas silagem de sorgo. As dietas tiveram uma relação de volumoso: concentrado de 40:60 em relação à matéria seca (MS) sendo isoprotéicas e isoenergéticas. Os animais foram abatidos aos 32 quilos de peso vivo. Antes do abate obtiveram-se as medidas biométricas, e logo após o mesmo, efetuou-se o peso de carcaça quente e as medidas morfométricas. Após 24 horas em câmara frigorífica à temperatura de $4{ }^{\circ} \mathrm{C}$, obteve- se o peso de carcaça fria e o cálculo do rendimento. A meia-carcaça esquerda foi dividida em cinco cortes: pescoço, paleta, pernil, costela e lombo, efetuandose o cálculo de rendimento dos cortes. As análises de qualidade de carne tais como $\mathrm{pH}$, cor $(\mathrm{L}, \mathrm{a}, \mathrm{b}$, croma e $\mathrm{H}^{\circ}$ ), perda de peso por cozimento, capacidade de retenção de água e força de cisalhamento foram realizadas em amostras do músculo Longissimus dorsi. O experimento foi conduzido em delineamento inteiramente casualizado. Para a análise de variância e o teste de Tukey para comparação das médias foi utilizado a probabilidade de 5\%. Não houve efeito de dietas ( $\mathrm{P}>0,05)$ de acordo com os parâmetros: desempenho produtivo, medidas corporais e qualidade de carne de cordeiros confinados. Também não houve efeito das dietas $(\mathrm{P}>0,05)$ sobre a área de olho do lombo admitindo-se que as carcaças mantiveram padrão semelhante de musculosidade fato importante para a aceitação de mercado. Concluise que, a substituição em até $75 \%$ da silagem de sorgo por resíduos de frutas (abacaxi, banana, manga e maracujá) na alimentação de cordeiros torna-se viável por não alterar o desempenho produtivo, as medidas corporais, os rendimentos de carcaça e cortes e características da carne de cordeiros confinados.

Palavras-chave: Alimentos alternativos, confinamento, ovinos, produção de carne
\end{abstract}

\footnotetext{
${ }^{1}$ Mestres em Zootecnia DZO, UFVJM, Diamantina, MG. E-mail: julio.ufvjm@hotmail.com; janazoo2003@gmail.com; janainaleite.zoo@gmail.com

2 Profs. Drs., Dept ${ }^{\circ}$ de Zootecnia. Universidade Federal dos Vales do Jequitinhonha e Mucuri, UFVJM, Diamantina, MG. E-mail: darcilenefigueiredo@yahoo.com.br; c.boari@ufvjm.edu.br

3 Pós-Doutora em Zootecnia, UFVJM, Diamantina, MG. E-mail: lopespaixao@yahoo.com.br

${ }^{4}$ Discente do Curso de Graduação em Zootecnia, UFVJM, Diamantina, MG. E-mail: marllucy.olicio@zootecnista.com.br

5 Discente do Curso de Mestrado em Zootecnia DZO, UFVJM, Diamantina, MG. E-mail: kariny_moreira@yahoo.com.br

* Autor para correspondência
} 


\begin{abstract}
This research was conducted with the objective to evaluate the use of residue dry matter (DM) from pineapple (Ananas comosus L.), banana (Musa sp.), mango (Mangifera indica) and passion fruit (Passiflora spp.) in feeding of the feedlot on productive performance, carcass yield and qualitative and quantitative characteristics of meat. Twenty-five crossbred lamps with Santa Inês breed and mixed breed were used. The treatments consisted of the replacement of $75 \%$ of sorghum silage by respective residue DM, whereas in the control treatment forage had only sorghum silage the diets had a houghageto-concentrate ratio of 40:60 interns of DM being isonitrogenous and isoenergetics. The animals were slaughtered at $32 \mathrm{~kg}$ liveweight. Before slaughter were obtained biometric measurements, after the same, was performed the hot carcasses weight and morphometric measurements. After 24 hours in a cold chamber at $4{ }^{\circ} \mathrm{C}$, was determined the cold carcass weight and yield calculation. The left half carcass was divided into five sections: neck, shoulder, shank, rib and loin, by performing the calculation of income cuts. Analyses meat quality such as $\mathrm{pH}$, color (L, a, b, chroma and Ho), by cooking weight loss, water retention capacity and shear strength were carried out in the Longissimus dorsi sample. The completely randomized design was adapted. The data were interpreted using analysis of variance with the test a Tukey 5\% probability. There was no effect of diet $(\mathrm{P}>0.05)$ according to the parameters: growth performance, body measurements, and meat quality of lambs. There was also no effect of the diets $(\mathrm{P}>0.05)$ on the loin eye area assuming that carcasses remained similar muscularity important fact to market acceptance standard. It is concluded that replacing up to $75 \%$ of sorghum silage by residues fruit (pineapple, banana, mango and passion fruit) in lambs feeding becomes feasible not to change the productive performance, body measurements, yields carcass and cuts and meat characteristics of feedlot lambs.
\end{abstract}

Key words: Alternative foods, feedlot, meat production, sheep

\section{Introdução}

A expansão das atividades da agroindústria processadora de frutas tem ocasionado grande quantidade de resíduos, os quais são produzidos de forma perene, pois, ao longo do ano, sempre há safra das mais diversas frutas. Dependendo da fruta que se utiliza, são gerados, como resíduos, casca, caroço, semente e bagaço (MATIAS et al., 2005).

A utilização destes coprodutos como matériaprima, além dos benefícios econômicos, representaria a redução de impactos ambientais e custos adicionais às empresas processadoras (LOUSADA JÚNIOR et al., 2005). Entretanto, na decorrência do desconhecimento de suas potencialidades ou de alternativas a sua utilização, parte considerável desta matéria prima é perdida ou utilizada de forma inadequada.

No semi-árido brasileiro se observa considerável expansão da agroindústria processadora de frutas, principalmente em localidades próximas a projetos de irrigação (LOUSADA JÚNIOR et al., 2006). Em muitas destas há, inclusive, relevante presença da ovinocaprinocultura.

A irregularidade na distribuição de chuvas, além dos baixos índices pluviométricos, têm sido limitantes à consolidação da ovinocultura no semiárido, principalmente durante estiagens, período com restrições na oferta de forragens (GONZAGA NETO et al., 2001). Por esta razão, propostas e tecnologias para o manejo nutricional, com custos reduzidos, devem ser apresentadas (CAPARRA et al., 2007).

Dentre as alternativas há de se considerar a utilização dos resíduos agroindustriais de frutas, dada à quantidade produzida, assim como a sua oferta, mesmo em períodos de estiagem. Entretanto, é fundamental o conhecimento sobre as variações em suas características nutricionais, as quais decorrem, dentre outros, da variedade das frutas, métodos de processamento e o tempo de armazenamento (LOUSADA JÚNIOR et al., 2006). Além disto, devem ser compreendidos os impactos 
sobre o desempenho animal e possíveis influências nas características da carne.

Como considerações, a utilização de resíduos da agroindústria processadora de frutas para alimentação de cordeiros pode representar alternativa interessante para a indústria e produtores. Para a agroindústria seria este um mecanismo para se reduzir custos operacionais do tratamento destes resíduos, bem como os procedimentos gerenciais a isto implicados. Por outro lado, para o produtor a utilização deste material pode ser uma alternativa para se reduzir os custos com a alimentação animal.

Para sistemas de produção instalados em regiões semiáridas, a utilização de algum destes resíduos seria uma forma de se minimizar a sazonalidade da oferta de forragem, tendo em vista a produção perene destes.

Entretanto, a utilização deste material tem algumas implicações práticas, para as quais os produtores devem se preparar, dentre elas os custos com transporte, necessidade de área para processo de secagem, gerenciamento da uniformidade da secagem (camadas de até $5 \mathrm{~cm}$ ) e controle de putrefação e proliferação de pragas.

Considerando-se o exposto, esta pesquisa foi conduzida com o objetivo de se avaliar o desempenho produtivo, medidas corporais, rendimento de carcaça e cortes e características da carne de ovinos alimentados com resíduos da agroindústria processadora de frutas, em substituição a silagem de sorgo.

\section{Material e Métodos}

A fase de confinamento do experimento foi conduzida na Fazenda Experimental do Moura, situada no município de Curvelo, Minas Gerais (18 45'21' 'Sul; 44²5'51' Oeste; 632m de altitude), durante o período de Setembro a Dezembro de 2011, totalizando 94 dias.
Para tal foram utilizados vinte e cinco cordeiros mestiços com Santa Inês e sem raça definida, machos, não castrados, com idade aproximada de oito meses e peso vivo inicial médio de $20,64 \pm 4,20$ $\mathrm{kg}$, os quais foram identificados individualmente com brincos na orelha e vermifugados antes do confinamento com medicamento à base de albendazole. Os cordeiros foram mantidos em baias individuais de $1,5 \mathrm{~m} \times 1,0 \mathrm{~m}$, equipadas com cocho, bebedouro e saleiros individuais.

O período experimental ( 84 dias) foi dividido em três fases de 28 dias cada. A pesquisa foi conduzida em delineamento inteiramente casualizado com cinco tratamentos diferenciados pelos seguintes resíduos: abacaxi, banana, manga e maracujá com cinco repetições. Do período experimental total, dos 10 dias foram destinados para adaptação às condições experimentais.

Os animais foram distribuídos balanceadamente e aleatoriamente em cinco tratamentos que consistiram da substituição de $75 \%$ do volumoso (silagem de sorgo) por resíduos de abacaxi (Ananas comosus L.), banana (Musa spp.), manga (Mangifera indica) e maracujá (Passiflora ssp.), sendo que no tratamento controle utilizou-se somente silagem de sorgo como volumoso (Tabela 1). Os animais receberam dietas contendo $60 \%$ de ração concentrada e $40 \%$ de volumoso, com base na matéria seca e com acesso ad libitum à água limpa e fresca.

O resíduo de abacaxi compunha-se por casca e polpa prensada, o de banana pela casca, o de manga por casca, caroço e polpa prensados e o de maracujá por casca e sementes. O resíduo de banana foi obtido em agroindústria localizada no município de Curvelo, Minas Gerais. Os resíduos de abacaxi, manga e maracujá foram obtidos em agroindústria instalada no município de Jaíba, Minas Gerais. A composição química dos resíduos da agroindústria processadora de sucos e da silagem de sorgo podem ser visualizados na Tabela 2.

Os resíduos foram secos ao sol, sobre lona preta, 
distribuídos em camada de, aproximadamente $5 \mathrm{~cm}$ Atingido aspecto de seco, em tato e visual, foram de espessura, sendo revolvidos três vezes ao dia. moídos em desintegrador com peneira $\mathrm{n}^{\circ} 1$.

Tabela 1. Composição percentual dos ingredientes (\% MS) nas rações fornecidas a cordeiros de corte.

\begin{tabular}{|c|c|c|c|c|c|}
\hline \multirow{2}{*}{ Ingredientes } & \multicolumn{5}{|c|}{ Tratamentos (\% MS) } \\
\hline & Abacaxi & Banana & Manga & Maracujá & Silagem de sorgo \\
\hline Milho fubá & 30 & 40 & 25 & 37 & 43 \\
\hline Farelo de Soja & 15 & 18,5 & 18,5 & 13 & 15 \\
\hline Farelo de trigo & 13,5 & - & 15 & 8,5 & - \\
\hline Ureia/SA (9:1) & - & - & - & - & 0,5 \\
\hline Mistura mineral $^{1}$ & 1,5 & 1,5 & 1,5 & 1,5 & 1,5 \\
\hline Resíduo de abacaxi & 30 & - & - & - & - \\
\hline Resíduo de banana & - & 30 & - & - & - \\
\hline Resíduo de manga & - & - & 30 & - & - \\
\hline Resíduo de maracujá & - & - & - & 30 & - \\
\hline Silagem de Sorgo & 10 & 10 & 10 & 10 & 40 \\
\hline
\end{tabular}

${ }^{1 /}$ Composição da mistura mineral (por quilograma): sódio - $147 \mathrm{~g}$; cálcio - $120 \mathrm{~g}$ fósforo - $87 \mathrm{~g}$; enxofre - $18 \mathrm{~g}$; zinco - $3.800 \mathrm{mg}$; ferro - $1.800 \mathrm{mg}$; manganês - 1.300 g; flúor - $870 \mathrm{mg}$; cobre - $590 \mathrm{mg}$; Mo = molibdênio - $300 \mathrm{mg}$; iodo - $80 \mathrm{mg}$; cobalto - $40 \mathrm{mg}$; cromo - $20 \mathrm{mg}$; selênio - $15 \mathrm{mg}$.

Fonte: Elaborada pelos autores.

Tabela 2. Composição química dos resíduos da agroindústria processadora de sucos e da silagem de sorgo

\begin{tabular}{lccccc}
\hline \multirow{2}{*}{\multicolumn{1}{c}{ Teor }} & \multicolumn{4}{c}{ Fontes volumosas } \\
\cline { 2 - 5 } & Abacaxi & Banana & Manga & Maracujá & Silagem de sorgo \\
\hline Matéria seca $^{*}$ Matéria orgânica $^{1}$ & 91,79 & 90,71 & 92,22 & 93,61 & 41,07 \\
Proteína brutaa $^{1}$ & 93,54 & 83,61 & 96,89 & 86,81 & 94,75 \\
NIDN $^{2}$ & 11 & 11,99 & 9,54 & 12,04 & 6,16 \\
NIDA $^{2}$ & 25,73 & 81,58 & 26,93 & 28,59 & 36,66 \\
Extrato Etéreo $^{1}$ & 6,83 & 14,06 & 6,38 & 10,18 & 9,78 \\
Cinza $^{1}$ & 2,21 & 7,46 & 4,71 & 3,8 & 2,57 \\
Carboidratos totais $^{1}$ & 6,46 & 16,39 & 3,11 & 13,19 & 5,28 \\
Fibra em detergente neutro - FDN $^{1}$ & 80,52 & 66,27 & 83,75 & 71,99 & 85,98 \\
FDN corrigida para cinza e proteína $^{1}$ & 50,63 & 44,31 & 33,68 & 53,43 & 57,47 \\
Carboidratos não fibrosos - CNF $^{1}$ & 46,1 & 35,11 & 25,49 & 52,04 & 52,82 \\
CNF corrigidos para cinza e proteína $^{1}$ & 28,72 & 20,18 & 48,96 & 13,85 & 28,51 \\
Fibra em detergente ácido - FDA $^{1}$ & 33,35 & 29,38 & 57,15 & 18,93 & 33,16 \\
Hemicelulose $^{1}$ & 27,08 & 23,61 & 20,76 & 38,77 & 28,11 \\
Celulose $^{1}$ & 23,55 & 20,7 & 12,92 & 14,66 & 29,36 \\
Lignina $^{1}$ & 23,29 & 14,94 & 15,48 & 24,53 & 23,5 \\
Nutrientes digestíveis totais - NDT $^{3}$ & 3,3 & 8,17 & 4,71 & 12,53 & 4,33 \\
\hline
\end{tabular}

${ }^{1 /}$ porcentagem de matéria seca. ${ }^{2 /} \mathrm{NIDIN}=$ nitrogênio insolúvel em detergente neutro (\% N total); NIDA = nitrogênio insolúvel em detergente ácido $(\% \mathrm{~N}$ total $) ;{ }^{3 /}$ NDT estimado segundo NRC $2001=($ FDNpd + PBd + EEd + CNFd $)-7$, DVCNF $=0,98(100$ $-[(\mathrm{FDN}-\mathrm{PIDN})+\mathrm{PB}+\mathrm{EE}+$ cinza $]) * \mathrm{FAP}, \mathrm{DVPB}($ forragens $)=\mathrm{PB} * \exp [-1,2 *(\mathrm{PIDA} / \mathrm{PB})], \mathrm{DVPB}($ concentrados $)=[1-(0,4 *$ $(\mathrm{PIDA} / \mathrm{PB}))^{*} \mathrm{~PB}, \mathrm{DVEE}=\mathrm{EE}-1, \mathrm{DVFDN}=0,75(\mathrm{FDN}-$ lignina $) *(1-\text { lignina/FDN })^{0,67}$

Fonte: Elaborada pelos autores. 
As rações foram formuladas para apresentar aproximadamente $17,32 \mathrm{~g} \cdot 100 \mathrm{~g}^{-1}$ de proteína bruta (PB) e $66,20 \mathrm{~g} \cdot 100 \mathrm{~g}^{-1}$ de nutrientes digestíveis totais (NDT) (Tabela 3), conforme recomendações do NRC (2007) para ovinos de $30 \mathrm{~kg}$ PV e ganho média diário de $200 \mathrm{~g} /$ dia. Estas foram fornecidas ad libitum, duas vezes ao dia, às $07 \mathrm{~h} 00$ e $15 \mathrm{~h} 00$ horas, permitindo-se sobras de, aproximadamente, $10 \%$.

O percentual de substituição da silagem pelo resíduo foi determinado com base no teor de fibra em detergente neutro da ração, em atenção aos limites mínimos requeridos à saúde ruminal, conforme NRC (2007) (Tabela 3).

Tabela 3. Composição química (\% MS) das rações fornecidas a cordeiros de corte.

\begin{tabular}{lccccc}
\hline \multirow{2}{*}{\multicolumn{1}{c}{ Teor }} & \multicolumn{4}{c}{ Tratamentos } \\
\cline { 2 - 5 } & Abacaxi & Banana & Manga & Maracujá & Silagem de sorgo \\
\hline Matéria seca $^{*} 86,47$ & 86,39 & 86,89 & 87,14 & 71,17 \\
Matéria orgânica $^{1}$ & 92,54 & 90,71 & 92,92 & 88,14 & 91,55 \\
Proteína bruta $^{1}$ & 16,88 & 17,46 & 18,07 & 16,92 & 17,27 \\
NIDN $^{2}$ & 12,65 & 28,82 & 13,43 & 14,88 & 16,68 \\
NIDA $^{2}$ & 6,83 & 14,61 & 6,38 & 10,18 & 9,78 \\
Extrato Etéreo $^{1}$ & 2,65 & 4,18 & 3,73 & 3,52 & 2,83 \\
Cinzas $^{1}$ & 7,46 & 9,29 & 7,09 & 11,87 & 8,46 \\
Carboidratos totais $^{1}$ & 73,07 & 69,69 & 71,44 & 67,99 & 71,44 \\
Fibra em detergente neutro - FDN $^{1}$ & 27,69 & 27,28 & 26,06 & 30,77 & 30,26 \\
FDN corrigida para cinza e proteína - & 21,88 & 19,16 & 17,89 & 26,02 & 24,31 \\
FDNcp $^{1}$ & 45,03 & 41,87 & 45,05 & 35,81 & 41,18 \\
Carboidratos não fibrosos - CNF $^{1}$ & 50,87 & 50 & 53,22 & 41,67 & 47,13 \\
CNF corrigidos para cinza e proteína - $_{\text {CNFcp }^{1}}$ & 13,15 & 11,63 & 11,85 & 16,78 & 13,41 \\
Fibra em detergente ácido - FDA $^{1}$ & 14,54 & 15,65 & 14,21 & 13,99 & 16,85 \\
Hemicelulose $^{1}$ & 10,95 & 7,9 & 9,13 & 11,32 & 10,92 \\
Celulose $^{1}$ & 2,03 & 3,56 & 2,53 & 4,92 & 2,38 \\
Lignina $^{1}$ &
\end{tabular}

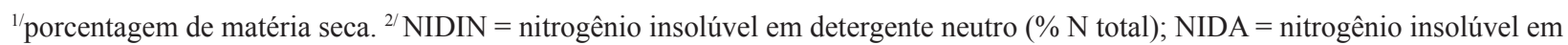
detergente acido ( $\% \mathrm{~N}$ total).

Fonte: Elaborada pelos autores.

A mistura de silagem, ração concentrada e dos resíduos foi realizada no momento da alimentação dos cordeiros.

Neste momento forneceu-se, também, mistura mineral, ad libitum, em cocho individualmente separado e instalado em cada baia.

Para avaliação de desempenho produtivo, os animais foram pesados no início do período experimental e a cada 15 dias, até que fosse atingido o peso de $32 \mathrm{~kg}$. O ganho médio diário (GMD) foi obtido pela diferença entre o PV final e inicial dividido pelo número de dias do experimento. Para a pesagem os cordeiros foram submetidos a jejum sólido de 16 horas. Avaliou-se o ganho médio diário (g/cab/dia), ganho total $(\mathrm{kg})$ e tempo médio, em dias, para alcançar $32 \mathrm{~kg}$ de PV cujo formula é (32 $\mathrm{kg}$ - Peso inicial)/GMD.

Atingido os $32 \mathrm{~kg}$, critério para o abate, os animais foram submetidos a jejum alimentar de 24 horas, havendo água potável a disposição. 
Finalizado o jejum, os cordeiros foram pesados (PV), sendo obtidas medidas biométricas, com uso de fita métrica de $1,50 \mathrm{~m}$, mantendo-se os animais em condições corretas de aprumo. Avaliouse o comprimento corporal, comprimento da perna anterior, comprimento da perna posterior, circunferência do tórax, profundidade do tórax e largura da garupa conforme Colomer Rocher, Delat e Sierra Alfranca (1988).

Os cordeiros foram insensibilizados por atordoamento mecânico não penetrativo, inspecionados quanto à eficiência da insensibilização (movimentação de globos oculares, respiração rítmica, vocalizações e movimentos coordenados) e abatidos por secção das artérias carótidas e veias jugulares, na entrada do peito. $\mathrm{Na}$ área suja procedeu-se à esfola, retirada de cabeça e patas. $\mathrm{Na}$ área limpa procedeu-se à evisceração e toalete das carcaças, utilizando-se água clorada (cloro residual $\left.50 \mathrm{mg} \cdot \mathrm{L}^{-1}\right)$, a $30^{\circ} \mathrm{C}$. As carcaças quentes inteiras foram pesadas para cálculo de rendimento, sendo este expresso como percentual do peso dos cordeiros ao abate. As caraças foram encaminhadas à câmara fria $\left(0^{\circ} \mathrm{C} \pm 1\right.$; umidade relativa $\left.85 \%\right)$, por 24 horas, penduradas pela articulação tarso-metatarsiana em ganchos distanciados em $17 \mathrm{~cm}$.

Após resfriamento as carcaças inteiras foram pesadas para cálculo de rendimento, sendo este expresso como percentual do peso ao abate e das carcaças a quente. Foram obtidas medidas morfométricas do comprimento interno da carcaça, profundidade do tórax, perímetro da garupa, comprimento externo da carcaça, comprimento da perna, largura da garupa e a largura do tórax, conforme Sañudo e Sierra (1986) e Garcia (1998). O índice de compacidade da carcaça foi determinado pela razão entre o peso da carcaça fria sobre o comprimento interno da carcaça. Em seguida as carcaças foram seccionadas em duas metades simétricas, por corte longitudinal ao longo da coluna vertebral, obtendo-se o peso da meia carcaça esquerda. A meia carcaça esquerda foi seccionada em pescoço, paleta, pernil e lombo, conforme Furusho-Garcia et al. (2004). Obteve-se o peso da costela inteira. Foram calculados, então, rendimentos dos cortes mencionados, os quais expressos como percentual do peso da meia carcaça esquerda resfriada.

Mensurou-se a área de olho de lombo (Longissimus dorsi), da meia-carcaça esquerda, à altura da $13^{\mathrm{a}}$ costela, por traçado de seu contorno em papel vegetal. Nos decalques foi elaborada uma escala $(\mathrm{cm})$, com régua milimétrica. As imagens foram escaneadas e analisadas em relação a sua área pelo programa Autocad. Os resultados foram expressos em $\mathrm{cm}^{2}$.

Avaliou-se características da carne proveniente da paleta, pernil, costela e lombo, obtidos $24 \mathrm{~h}$ após o início do resfriamento.

A mensuração do $\mathrm{pH}$ foi feita por uso de $\mathrm{pHmetro}$ acoplado por eletrodo de penetração (Hanna Instruments) sendo os valores de cada repetição calculados a partir da média de 3 leituras.

A cor foi realizada utilizando colorímetro Chroma Meter CR-400 (Konica Minolta, Japão), empregando iluminante D65 e geometria 45/0, e os valores de cor foram expressos no sistema CIELAB. Os valores de $\mathrm{L}^{*}$ (luminosidade), a* (componente vermelho-verde) $\mathrm{e}^{*}$ (componente amarelo-azul) foram obtidos diretamente do colorímetro calibrado para um padrão branco em ladrilho e utilizados para cálculo da tonalidade cromática $\mathrm{H}^{\mathrm{o}}\left(\mathrm{H}^{\circ}=\arctan \mathrm{b}^{*} / \mathrm{a}^{*}\right)$ e croma $\left[\mathrm{C}^{*}=\right.$ $\left.\left(a^{* 2}+b^{* 2}\right)^{1 / 2}\right] . L^{*}$ varia de 0 a 100 , em que o valor 0 indica o preto (ou cor escura) e o 100, o branco (cor clara). Para $\mathrm{H}^{*}$; o 0 representa vermelho puro; o 90, o amarelo puro; o 180, o verde puro; e o 270, o azul puro. Valores de $\mathrm{H}^{\circ}$ próximos de 90 , indicam tonalidade amarela, e, quanto mais próximos de 0 , a tonalidade vermelha. Com relação ao croma, quanto mais altos os valores de $\mathrm{C}^{*}$; mais viva a cor observada (LAWLESS; HEYMANN, 1998). Os 
valores de cada repetição foram calculados a partir da média de 3 leituras.

A capacidade de retenção de água (CRA\%) foi determinada pela disposição de frações de $0,5 \mathrm{~g}$ de carne entre dois papéis filtro e estes entre duas placas de vidro $(12 \times 12 \times 1 \mathrm{~cm})$, sob peso de $10 \mathrm{~kg}$ por 5 min (RAMOS; GOMIDE, 2007). A capacidade de retenção de água foi obtida pela relação entre peso final e inicial das amostras, sendo o resultado expresso em percentagem.

A perda de peso por cocção (PPC\%) foi determinada por aquecimento, em chapa metálica com dupla face (regulada a $150^{\circ} \mathrm{C}$ ), de porção de + ou - 30g de carne embalada em folha metalizada por tempo suficiente para assegurar temperatura interna dentre 82 a $85^{\circ} \mathrm{C}$ (RAMOS; GOMIDE, 2007). A perda de peso por cocção foi calculada em função da diferença entre o peso inicial e final, sendo expressa em percentagem.

A força de cisalhamento foi determinada por texturômetroTA.XT2 plus ${ }^{\circledR}$ Stable Micro Systems acoplado com sonda Warner-Bratzler, com espessura de $1,016 \mathrm{~mm}$ e com lâmina de $3,05 \mathrm{~mm}$. Os dados (picos positivos máximos) foram obtidos empregando-se o programa Exponent Lite versão 5.1 (Stable Micro Systems). O equipamento foi calibrado com peso-padrão de $5 \mathrm{~kg}$ e a velocidade de descida e corte do dispositivo foi de $200 \mathrm{~mm} /$ minuto. Para as análises foram usadas das amostras remanescentes da análise da perda de peso por cozimento, nas quais foram retiradas sub-amostras em forma de paralelepípedos $1 \times 1 \times 2 \mathrm{~cm}$ (altura, largura e comprimento, respectivamente), sendo dispostas no equipamento com as fibras orientadas no sentido perpendicular a lâmina. Os resultados foram expressos em $\mathrm{Kgf} / \mathrm{cm}^{2}$ (AMSA, 1995).
Os dados foram interpretados por análise de variância e teste de Tukey, em nível de probabilidade de 5\%, empregando-se o programa Statistical Analysis System (SAS, 2002), sendo considerado 2 parcelas perdidas nos tratamento com resíduos de abacaxi e de maracujá.

\section{Resultados e Discussão}

No decorrer do experimento um animal do tratamento abacaxi e outro no tratamento maracujá, morreram, acometidos por doença respiratória. Não houve efeito $(\mathrm{P}>0,05)$ das diferentes tratamentos sobre o ganho médio diário e dias para atingir 32 kg (Tabela 4). Considerando-se a padronização em peso inicial e peso final, justifica-se, portanto, a variação não significativa sobre o ganho médio total.

A semelhança dos ganhos médios diários pode ser devido a aproveitamento ruminal e metabólico dos nutrientes dos tratamentos, visto que os mesmos apresentaram valores semelhantes e termos nutricionais como mostra a Tabela 3.

Alves et al. (2003) menciona que dietas em diferentes níveis energéticos e protéicos poderiam influenciar o ganho médio diário e ganho total, fato não observado nesta pesquisa, na decorrência das rações propostas serem isoenergéticas e isoprotéicas.

Também avaliando resíduo de frutas, Ferreira et al. (2009) obtiveram médias de ganho de peso semelhantes ao do presente trabalho, quando avaliaram a adição de subprodutos de acerola, caju e abacaxi à silagem de capim-elefante, sendo 123,$2 ; 154,4$ e $164,8 \mathrm{~g} /$ animal/dia os ganhos para os respectivos tratamentos. 
Tabela 4. Desempenho produtivo de cordeiros alimentados com resíduos da agroindústria processadora de frutas

\begin{tabular}{lcccccc}
\hline \multirow{2}{*}{\multicolumn{1}{c}{ Variável }} & \multicolumn{7}{c}{ Tratamentos $^{1}$} \\
\cline { 2 - 7 } & Abacaxi & Banana & Manga & Maracujá & Silagem de sorgo & $\mathrm{CV}(\%)^{2}$ \\
\hline Peso médio inicial & 20,23 & 20,25 & 20,27 & 20,29 & 20,29 & 22,8 \\
Peso médio final & 31,87 & 32,43 & 32,12 & 32,57 & 31,40 & 7,5 \\
Ganho médio diário (g/cab/dia) & 169,50 & 175,40 & 171,20 & 171,75 & 152,00 & 28,1 \\
Dias para alcançar 32 kg & 63 & 72 & 75 & 65 & 77 & 39,4 \\
\hline
\end{tabular}

1/ Médias seguidas por letras diferentes na mesma linha diferem entre si $(\mathrm{P}<0,05)$ pelo teste Tukey. ${ }^{2} \mathrm{CV}=$ coeficiente de variação. Fonte: Elaborada pelos autores.

A variável, dias para atingir $32 \mathrm{~kg}$ apresentou uma situação favorável em relação aos alimentos alternativos, demonstrando médias numericamente inferiores para os tratamentos com utilização de resíduos em relação ao uso de silagem de sorgo como única fonte de volumoso. De acordo com o presente estudo, se considerarmos a média dos tratamentos com resíduos em relação ao tratamento controle, verifica-se menos dias para manter os animais em confinamento. Isto representa menos custos associados à manutenção dos animais, bem como giro mais rápido de capital haja vista a diminuição de dias no ciclo de produção de animais alimentados com os resíduos. Apesar de não apresentar diferença $(\mathrm{P}>0,05)$ nos tratamentos abacaxi e maracujá a variável dias para atingir $32 \mathrm{~kg}$ apresentaram os menores valores em relação aos outros resíduos.

A dieta não influenciou significativamente as medidas biométricas, morfométricas e a área de olho do lombo (Tabela 5) nos animais.

Tabelas 5. Médias das características biométricas in vivo e morfométricas de cordeiros alimentados com resíduos da agroindústria processadora de frutas.

\begin{tabular}{lcccccc}
\hline \multirow{2}{*}{ Parâmetro } & \multicolumn{7}{c}{ Tratamentos } \\
\cline { 2 - 6 } & Abacaxi & Banana & Manga & Maracujá & Silagem de sorgo & CV $(\%)$ \\
\hline C. corporal (cm) & 43,00 & 41,80 & 43,00 & 46,38 & 41,80 & 10,3 \\
C. P. anterior (cm) & 63,50 & 62,50 & 62,90 & 62,00 & 64,00 & 7,2 \\
C.P. posterior (cm) & 67,40 & 66,20 & 66,75 & 65,25 & 65,20 & 4,8 \\
Circ. tórax (cm) & 75,00 & 75,70 & 73,90 & 75,25 & 74,60 & 3,2 \\
Prof. tórax (cm) & 26,50 & 27,80 & 26,60 & 27,25 & 27,98 & 8,1 \\
L. garupa (cm) & 21,38 & 21,10 & 21,70 & 22,63 & 20,60 & 6,0 \\
\hline C. C. externo (cm) & 42,50 & 43,90 & 42,80 & 45,75 & 43,60 & 7,1 \\
C. interno (cm) & 52,25 & 44,60 & 47,50 & 49,38 & 51,76 & 8,1 \\
C. perna $(\mathrm{cm})$ & 23,25 & 23,40 & 23,96 & 23,50 & 22,90 & 7,8 \\
L. garupa (cm) & 20,27 & 20,44 & 20,34 & 20,80 & 20,88 & 7,1 \\
Per. garupa (cm) & 54,63 & 56,56 & 56,84 & 58,50 & 58,00 & 6,6 \\
AOL $\left(\mathrm{cm}^{2}\right)$ & 16,92 & 15,87 & 14,99 & 16,15 & 14,92 & 19,5 \\
\hline
\end{tabular}

C. corporal $=$ comprimento corporal; C. P. anterior $=$ comprimento de perna anterior; C. P. posterior= comprimento de perna posterior; Circ. tórax = circunferência de tórax; Prof. tórax= profundidade de tórax; L. garupa= largura de garupa; $\mathrm{C}$. C. externo = comprimento carcaça externo; $\mathrm{C}$. interno= comprimento interno; C. perna = comprimento de perna; L. garupa= largura de garupa; Per. garupa $=$ perímetro de garupa; Prof. tórax $=$ profundidade de tórax; $\mathrm{AOL}=$ Área de olho de lombo; $\mathrm{cm}^{=} \mathrm{centímetros} ; \mathrm{cm}^{2}=$ centímetros quadrados. $\mathrm{CV}=$ Coeficiente de variação das médias. Médias seguidas pela mesma letra nas linhas não diferem entre si pelo teste de Tukey em probabilidade de $5 \%$.

Fonte: Elaborada pelos autores. 
Por esta razão, admite-se que a substituição de $75 \%$ da fração volumosa pelos resíduos estudados não interferiu no padrão das carcaças, sendo importante para sua aceitação de mercado (XENOFONTE et al., 2009).

De forma semelhante, Araújo Filho et al. (2007) não detectaram efeito significativo da dieta sobre medidas morfométricas de ovinos confinados, exceto para a circunferência do tórax e largura do peito, o qual atribuem a maior deposição de tecidos observada em dietas de maior energia, o que não foi observado neste estudo, haja vista as dietas apresentarem teores semelhantes de NDT.

Garcia et al. (2000) não observaram efeito significativo da utilização da casca de café sobre medidas biométricas de cordeiros, exceto para comprimento de perna e profundidade do tórax. Segundo estes autores tais diferenças foram acarretadas pela menordisponibilidadedenutrientes da casca, a qual apresenta grande quantidade de lignina, afetando, portanto, o crescimento ósseo, sobretudo de regiões anatômicas mais sensíveis, como tórax e perna. Sobre isto, admite-se que os resíduos de abacaxi (lignina 3,3g.100 $\mathrm{g}^{-1}$ ), banana (lignina $8,17 \mathrm{~g} .100 \mathrm{~g}^{-1}$ ), manga (lignina 4,71g. $100 \mathrm{~g}^{-1}$ ) e maracujá (lignina $3,3 \mathrm{~g} \cdot 100 \mathrm{~g}^{-1}$ ) não tenham oferecido este tipo de empecilho ao desenvolvimento ponderal, tendo em vista que as medidas biométricas e morfométricas em animais alimentados com resíduos foram semelhantes àquelas observadas em cordeiros exclusivamente alimentados com sorgo (lignina 4,33g.100 g-1), enquanto fração volumosa.

Medidas biométricas e morfométricas, assim como índices zootécnicos e desempenho são importantes à caracterização fenotípica de grupos raciais, indispensável ao melhoramento do plantel, ao conhecimento da conformação dos indivíduos, ao estabelecimento da relação entre conformação e funcionalidade e definição de porte e aptidão (SOUSA et al., 2009; ARAÚJO FILHO et al. 2007;
PINHEIRO; JORGE, 2010 ).

A área de olho de lombo (Longissimus dorsi) é considerada como medida representativa da musculosidade de carcaças, e, por esta razão, têm sido parâmetro para classificações e avaliação de sua qualidade e rendimento (ALVES et al., 2003; HASHIMOTO et al., 2012). Não havendo efeito $(\mathrm{P}>0,05)$ das dietas sobre a área de olho do lombo admite-se que as carcaças mantiveram padrão semelhante de musculosidade. Tal fato se verifica pelo efeito não significativo das dietas sobre rendimentos de carcaça e cortes e índice de compacidade (Tabela 6).

Rendimentos de carcaça a quente e fria obtidos nesta pesquisa mantiveram semelhança com dados relatados por Silva et al. (2011) que também trabalharam com resíduo de fruta. Cunha et al. (2001) relatam efeito não significativo do uso de diferentes volumosos sobre a área de olho de lombo, rendimentos de carcaça, quente e fria, e cortes de cordeiros confinados. Costa et al. (2011) também não observaram efeito significativo da utilização de fruto-refugo de melão na dieta de cordeiros confinados, sobre rendimento de carcaça, quente e fria, área de olho de lombo e cortes comerciais (pescoço, costela, paleta, lombo e a perna). Esses autores atribuíram essa semelhança à lei da harmonia anatômica (BOCCARD; DUMONT, 1960) de que carcaças com pesos e concentrações de gordura similares apresentam praticamente todas as regiões corporais em proporções semelhantes, independentemente da conformação dos genótipos considerados.

Os rendimentos de pernil, lombo e paleta obtidos nesta pesquisa não foram influenciados significativamente pelos tratamentos o que implica que os resíduos em estudos podem ser utilizados sem comprometer as partes com maior rendimento comestível das carcaças (SILVA SOBRINHO et al., 2005). 
Tabela 6. Peso e rendimento de carcaça e cortes comerciais de cordeiros alimentados com resíduos da agroindústria processadora de frutas.

\begin{tabular}{lcccccc}
\hline \multirow{2}{*}{ Parâmetro } & \multicolumn{7}{c}{ Tratamentos } \\
\cline { 2 - 7 } & Abacaxi & Banana & Manga & Maracujá & Silagem de sorgo & CV(\%) \\
\hline PCQ (Kg) & 14,77 & 14,92 & 14,22 & 15,79 & 14,06 & 7,7 \\
PCF (Kg) & 14,60 & 14,67 & 14,01 & 15,61 & 13,85 & 7,7 \\
ICC (kg/cm) & 0,28 & 0,33 & 0,30 & 0,32 & 0,27 & 12,5 \\
RCQ (\%) & 46,37 & 46,03 & 44,27 & 48,45 & 44,89 & 4,9 \\
RCF (\%) & 45,85 & 45,32 & 43,63 & 47,91 & 44,22 & 5,4 \\
RCF* (\%) & 98,88 & 98,41 & 98,54 & 98,81 & 98,53 & 1,1 \\
R. Pescoço (\%) & 5,82 & 4,92 & 6,06 & 5,76 & 6,01 & 20,1 \\
R. Paleta (\%) & 15,91 & 16,28 & 15,45 & 14,77 & 14,84 & 7,2 \\
R. Pernil (\%) & 27,73 & 26,69 & 27,83 & 27,83 & 26,87 & 4,4 \\
R. Costela (\%) & 36,19 & 37,05 & 36,43 & 38,07 & 35,70 & 7,6 \\
R. Lombo (\%) & 6,14 & 7,18 & 5,59 & 5,98 & 7,42 & 16,3 \\
\hline
\end{tabular}

$\mathrm{PCQ}=$ peso carcaça quente; $\mathrm{PCF}=$ peso carcaça fria; $\mathrm{ICC}=$ Índice de compacidade da carcaça; $\mathrm{RCQ}=$ rendimento carcaça quente; $\mathrm{RCQ}=$ rendimento carcaça fria; * Rendimento em função do PCQ. R. Pescoço= rendimento pescoço; R. Paleta= rendimento paleta; R. Pernil= rendimento pernil; R. Costela $=$ rendimento costela; R. Lombo $=$ rendimento lombo; $(\mathrm{Kg})=$ kilograma; $(\mathrm{Kg} / \mathrm{cm})=$ kilograma /centímetro; $(\%)=$ porcentagem. $\mathrm{CV}=$ Coeficiente de variação das médias. Médias seguidas pela mesma letra nas linhas não diferem entre si pelo teste de Tukey em probabilidade de 5\%.

Fonte: Elaborada pelos autores.

As dietas não influenciaram $(\mathrm{P}<0,05)$ a compacidade das carcaças, tendo em vista que não promoveram alterações significativas no peso da carcaça a frio e no comprimento interno da carcaça. Conforme Soares et al. (2012) e Cartaxo et al. (2011) variações nos teores energéticos e protéicos das dietas promovem alterações nos pesos de carcaça fria e, consequentemente, elevam o índice de compacidade da carcaça, em animais semelhantes. Esta variação, no entanto, não foi observada nesta pesquisa, para a qual as formulações foram feitas pra proporcionar o mesmo consumo diário de NDT aos animais. É importante enfatizar que a compacidade da carcaça está estreitamente vinculada conformação da carcaça e quanto melhor a conformação muscular da carcaça maior é o índice de compacidade. Os índices de compacidade encontrados nesta pesquisa indicaram boa proporção de tecido muscular por unidade de comprimento se comparados aos valores obtidos em pesquisas com condições e genótipos distintos. Valores esse que se encontram entre 0,15 a $0,28 \mathrm{~kg} /$ cm (CUNHA et al., 2000; SIQUEIRA; SIMÕES;
FERNANDES, 2001; PINHEIRO; JORGE, 2010; COSTA et al., 2011; SILVA et al., 2012).

Não houve efeito $(\mathrm{P}>0,05)$ das dietas sobre as características da carne proveniente dos cortes paleta, pernil, costela e lombo (Tabela 7).

A mensuração do $\mathrm{pH}$ no músculo L. dorsi, 24 horas após o abate, é reconhecida como um dos principais indicativos do manejo humanitário ante mortem e, também, do processo normal e desejável de conversão do músculo em carne (COSTA et al., 2011; DEVINE et al., 1983).

Não houve efeito significativo das dietas sobre o $\mathrm{pH}_{24 \text { horas }}$ do lombo, bem como da paleta, pernil e costela (Tabela 7). Os valores médios estiveram dentre o intervalo desejável de 5,5 a 5,85, no qual se descreve a carne vermelho-cereja, de consistência firme e não exsudativa (GONÇALVES et al., 2004; SILVA SOBRINHO et al., 2005; BATISTA, 2008).

Os dados para $\mathrm{pH}_{24 \text { horas }}$ desta pesquisa mantém semelhança com resultados publicados na literatura (SCERRA et al., 2001; ZEOLA et al., 2001; OLFAZ et al., 2005; CAPARRA et al., 2007). 
Tabela 7. Características da carne da paleta, pernil, costa e lombo de cordeiros alimentados com resíduos da agroindústria processadora de frutas.

\begin{tabular}{|c|c|c|c|c|c|c|}
\hline \multirow{2}{*}{ Paleta } & \multicolumn{5}{|c|}{ Tratamento } & \multirow[b]{2}{*}{$\mathrm{CV}(\%)$} \\
\hline & Abacaxi & Banana & Manga & Maracujá & Silagem de sorgo & \\
\hline $\mathrm{pH}$ & 5,73 & 5,66 & 5,71 & 5,68 & 5,65 & 4,5 \\
\hline $\mathrm{L}^{*}$ & 43,18 & 45,66 & 45,66 & 45,27 & 45,00 & 6,2 \\
\hline$a^{*}$ & 17,50 & 16,61 & 18,17 & 17,10 & 15,64 & 10,4 \\
\hline$b^{*}$ & 9,18 & 9,29 & 9,50 & 9,52 & 7,04 & 30,3 \\
\hline Croma & 19,82 & 19,13 & 20,59 & 19,92 & 17,20 & 12,5 \\
\hline $\mathrm{H}^{\circ}$ & 27,17 & 28,97 & 27,29 & 30,39 & 23,98 & 22,4 \\
\hline PPC (\%) & 34,66 & 33,54 & 33,99 & 37,79 & 34,98 & 8,7 \\
\hline CRA $(\%)$ & 69,04 & 64,70 & 67,85 & 67,66 & 65,53 & 10,2 \\
\hline $\mathrm{FC}(\mathrm{KgF})$ & 4,47 & 4,16 & 4,32 & 4,16 & 3,46 & 43,4 \\
\hline \multirow{2}{*}{ Pernil } & \multicolumn{5}{|c|}{ Tratamento } & \\
\hline & Abacaxi & Banana & Manga & Maracujá & Silagem de sorgo & $\mathrm{CV}(\%)$ \\
\hline $\mathrm{pH}$ & 5,62 & 5,73 & 5,68 & 5,62 & 5,71 & 4,9 \\
\hline $\mathrm{L}^{*}$ & 41,00 & 44,32 & 42,07 & 40,29 & 43,01 & 6,3 \\
\hline$a^{*}$ & 17,39 & 16,29 & 18,62 & 17,78 & 16,88 & 7,9 \\
\hline $\mathrm{b}^{*}$ & 7,98 & 9,83 & 8,71 & 9,00 & 7,53 & 27,7 \\
\hline Croma & 19,24 & 19,12 & 20,63 & 20,00 & 18,55 & 10,6 \\
\hline $\mathrm{H}^{\circ}$ & 23,92 & 30,76 & 25,20 & 26,61 & 23,81 & 20,8 \\
\hline PPC (\%) & 40,09 & 36,47 & 37,72 & 37,05 & 37,27 & 9,0 \\
\hline CRA (\%) & 63,06 & 62,81 & 63,68 & 59,48 & 66,50 & 15,3 \\
\hline $\mathrm{FC}(\mathrm{KgF})$ & 5,54 & 4,44 & 6,15 & 6,56 & 6,74 & 47,4 \\
\hline \multirow{2}{*}{ Costela } & \multicolumn{5}{|c|}{ Tratamento } & \\
\hline & Abacaxi & Banana & Manga & Maracujá & Silagem de sorgo & $\mathrm{CV}(\%)$ \\
\hline $\mathrm{pH}$ & 5,72 & 6,05 & 5,74 & 5,74 & 6,03 & 4,2 \\
\hline $\mathrm{L}^{*}$ & 41,74 & 45,40 & 47,23 & 45,39 & 43,84 & 9,4 \\
\hline$a^{*}$ & 19,35 & 17,95 & 17,60 & 17,27 & 17,39 & 10,6 \\
\hline $\mathrm{b}^{*}$ & 8,88 & 9,79 & 9,67 & 10,75 & 7,02 & 28,0 \\
\hline Croma & 21,32 & 20,57 & 20,17 & 20,39 & 18,80 & 12,7 \\
\hline $\mathrm{H}^{\circ}$ & 24,43 & 28,44 & 28,06 & 31,68 & 21,86 & 19,9 \\
\hline PPC (\%) & 52,33 & 34,04 & 31,97 & 52,02 & 38,85 & 28,6 \\
\hline CRA (\%) & 47,75 & 61,16 & 60,75 & 51,98 & 58,98 & 22,0 \\
\hline $\mathrm{FC}(\mathrm{KgF})$ & 4,25 & 4,55 & 5,06 & 4,16 & 4,15 & 27,9 \\
\hline \multirow{2}{*}{ Lombo } & \multicolumn{5}{|c|}{ Tratamento } & \\
\hline & Abacaxi & Banana & Manga & Maracujá & Silagem de sorgo & $\mathrm{CV}(\%)$ \\
\hline $\mathrm{pH}$ & 5,49 & 5,65 & 5,62 & 5,59 & 5,58 & 6,5 \\
\hline $\mathrm{L}^{*}$ & 39,13 & 41,27 & 40,16 & 41,70 & 41,07 & 9,6 \\
\hline$a^{*}$ & 17,34 & 15,62 & 16,67 & 17,08 & 16,46 & 14,1 \\
\hline $\mathrm{b}^{*}$ & 7,22 & 9,01 & 8,74 & 9,21 & 9,21 & 24,2 \\
\hline Croma & 18,80 & 18,21 & 18,98 & 19,51 & 19,09 & 11,2 \\
\hline $\mathrm{H}^{\circ}$ & 22,33 & 29,58 & 27,62 & 28,46 & 29,86 & 26,3 \\
\hline PPC (\%) & 34,38 & 38,22 & 39,25 & 48,02 & 41,02 & 9,6 \\
\hline CRA (\%) & 63,22 & 60,26 & 60,34 & 62,27 & 59,31 & 8,1 \\
\hline $\mathrm{FC}(\mathrm{KgF})$ & 4,84 & 3,71 & 4,34 & 5,00 & 4,99 & 36,3 \\
\hline
\end{tabular}

$\mathrm{pH}=$ Potencial hidrogeniônico; $\mathrm{L}^{*}=$ luminosidade; $\mathrm{a}^{*}=$ componente vermelho-verde; $\mathrm{b} *$ componente amarelo-azul; $; \mathrm{H}^{\circ}=$ tonalidade cromática; $\mathrm{PPC}=$ perda por cocção; $\mathrm{CRA}=$ capacidade de retenção de água; $\mathrm{FC}=$ força de cisalhamento; $(\%)=$ porcentagem; $\mathrm{KgF}=$ kilograma/força. $\mathrm{CV}=$ Coeficiente de variação das médias. Médias seguidas pela mesma letra nas linhas não diferem entre si pelo teste de Tukey em probabilidade de 5\%.

Fonte: Elaborada pelos autores. 
A luminosidade $\left(\mathrm{L}^{*}\right)$, teor de vermelho $\left(a^{*}\right)$, teor de amarelo $\left(b^{*}\right)$ e intensidade da cor (croma) e tonalidade $\left(\mathrm{H}^{\circ}\right)$ da carne não foram significativamente influenciados pelas dietas pesquisadas (Tabela 7). Scerra et al. (2001) e Russo et al. (1999) também não observaram significância de diferentes sobre a cromaticidade da carne de cordeiros.

$\mathrm{Na}$ literatura são apresentadas variações da luminosidade ( $\mathrm{L}^{*}$ ) de 31,36 a 45,61 e de 12,27 a 17,06 para o teor de vermelho (a*) e de 3,34 a 6,51 para o teor de amarelo (b*) (RUSSO et al., 1999; BRESSAN et al., 2001; BONAGURIO et al., 2003; MADRUGA et al., 2005), valores dos quais se aproximam aqueles obtidos nesta pesquisa e que se encontram dentro do desejado para a carne ovina.

Não houve efeito $(\mathrm{P}>0,05)$ da substituição da silagem de sorgo pelos resíduos do processamento de frutas sobre a capacidade de retenção de água e perda de peso por cozimento da carne dos cordeiros (Tabela 7), parâmetros estes que contribuem à compreensão do rendimento da carne durante o seu preparo para o consumo. Huff-Lonergan e Lonergan (2005) mencionam a importância da capacidade de retenção de água sobre a cor e luminosidade da carne, os quais são dependentes da quantidade de luz incidente absorvida ou refletida, em função da água na matriz cárnea. Os valores obtidos nesta pesquisa se assemelham àqueles disponíveis na literatura (SANTOS-SILVA; BESSA; MENDES, 2003; ZEOLA et al., 2007; RODRIGUES et al., 2008).

Por intermédio da análise instrumental da força de cisalhamento se avalia a maciez da carne, um dos principais parâmetros a sua aceitação. Não foram encontradas diferenças $(\mathrm{P}>0,05)$ das dietas sobre a força de cisalhamento da carne dos cortes pesquisados (Tabela 7). Pinheiro et al. (2009) trabalhando com cordeiros confinados recebendo diferentes relações de volumoso:concentrado não obtiveram, também, efeito significativo das diferentes dietas sobre a força de cisalhamento da carne de cordeiros. Estes autores mencionam que este parâmetro seria mais influenciado por fatores que afetam o sistema enzimático e a quantidade energética presentes nas fibras musculares. Além destes fatores, Silva Sobrinho et al. (2005) e Rota et al. (2006) mencionam também a idade ao abate como influenciador da maciez da carne de cordeiros.

O tecido conectivo que confere resistência ao músculo e aos tendões é constituído principalmente pelo colágeno. Segundo Cranwell et al. (1996), o colágeno total é relativamente constante nos animais nas diferentes idades, mas, com o aumento da idade, as ligações tornam-se mais resistentes e estáveis, conferindo à carne maior resistência ao corte.

Mesmo apresentando força de cisalhamento maiores devido a idade ao abate dos animais, baseado na escala proposta por Bickerstaffe et al. (2001) ao estratificar a textura da carne de lombo de cordeiros utilizando dados gerados por análise sensorial e mensuração instrumental da força de cisalhamento as médias para força de cisalhamento obtidas nesta pesquisa se enquadram na categoria de carne muito macia, cuja força de cisalhamento é inferior a $5 \mathrm{kgF}$.

\section{Conclusão}

A substituição no fornecimento da matéria seca da silagem de sorgo pode ser feita em até $75 \%$ por resíduos gerados pelo processamento agroindustrial de abacaxi, banana, manga e maracujá sem alterar o desempenho produtivo, as medidas corporais, os rendimentos de carcaça e cortes e características da carne de cordeiros confinados.

\section{Agradecimentos}

Os autores agradecem ao CNPq, CAPES, FINEP, FAPEMIG, UFVJM, Pomar Brasil Agroindustrial ${ }^{\circ}$.

Registro em Comitê de Ética com pesquisa envolvendo animais: $\mathrm{CEP} / \mathrm{N}^{\circ} 018 / 11 / \mathrm{UFVJM}$. 


\section{Referências}

ALVES, K. S.; CARVALHO, F. F. R.; VERAS, A. S. C.; FERREIRA, M. A.; COSTA, R. G.; SANTOS, E. P.; FREITAS, R. G.; SANTOS JÚNIOR, C. M.; ANDRADE, D. K. B. Níveis de energia em dietas para ovinos Santa Inês: Digestibilidade Aparente. Revista Brasileira de Zootecnia, Viçosa, MG, v. 32, n. 6, p. 1962-1968, 2003.

AMERICAN meat science association-AMSA. Research guidelines for cookery, sensory evaluation and tenderness measurements of fresh meat. Natl. LiveStock and Meat Board, Chicago, IL, 1995.

ARAÚJO FILHO, J. T.; COSTA, R. G.; FRAGA, A. B.; SOUSA, W. H.; GONZAGA NETO, S.; BATISTA, A. S. M.; CUNHA, M. G. G. Efeito de dieta e genótipo sobre medidas morfométricas e não constituintes da carcaça de cordeiros deslanados terminados em confinamento. Revista Brasileira de Saúde e Produção Animal, Bahia, v. 8, n. 4, p. 394-404, out./dez. 2007.

BATISTA, A. S. M. Qualidade da carne de ovinos morada nova, Santa Inês e Mestiços Dorper x Santa Inês submetidos a dietas com diferentes concentrações energéticas. 20008. PhD Tese (Programa de Doutorado Integrado em Zootecnia) - Universidade Federal da Paraíba, Areia.

BICKERSTAFFE, R.; BEKHIT, A. E. D.; ROBERTSON, L. J.; ROBERTS, N.; GEESINK, G. H. Impact of introducing specifications on the tenderness of retail meat Original Research Article. Meat Science, Barking, v. 59, n. 3, p. 303-315, nov. 2001.

BOCCARD, R.; DUMONT, B. L. Etude de la production de la viande chez les ovins. II. Variation de l'importance relative dês diferentes régions corporelles de l'agneau de boucherie. Annales de Zootechnie, França, v. 9, n. 4, p. 355-365, 1960.

BONAGURIO, S.; PÉREZ J. R. O.; FURUSHO GARCIA, I. F.; BRESSAN, M. C.; LEMOS, A. L. S. Qualidade da carne de cordeiros Santa Inês puros e mestiços com texel abatidos com diferentes pesos. Revista Brasileira de Zootecnia, Viçosa, MG, v. 32, n. 6, p. 1981-1991, 2003.

BRESSAN, C.; PRADO, O. V.; PÉREZ, J. R. O.; LEMOS, A. L. S. C. Efeito do peso ao abate de cordeiros Santa Inês e Bergamácia sobre as características físicoquímicas da carne. Ciência e Tecnologia de Alimentos, Campinas, v. 21, n. 3, p. 293-303, 2001.

CAPARRA, P.; FOTI, F.; SCERRA, M.; SINATRA, M. C.; SCERRA, V. Solar-dried citrus pulp as an alternative energy source in lamb diets: effects on growth an carcass and meat quality. Small Ruminant Research, Amsterdam, v. 40, n. 3, p. 303-311, 2007.
CARTAXO, F. Q.; SOUSA, W. H.; COSTA, R. G.; CEZAR, M. F.; PEREIRA FILHO, J. M.; CUNHA, M. G. G. Características quantitativas da carcaça de cordeiros de diferentes genótipos submetidos a duas dietas. Revista Brasileira de Zootecnia, Viçosa, MG, v. 40, n. 10, p. 2220-2227, 2011.

COLOMER ROCHER, F.; MORAND-FEHR, P.; KIRTON, A. H.; DELFA BELENGUER, R.; SIERRA A. I. Métodos normalizados para el estudio de los caracteres cuantitativos y cualitativos de las canales caprinas y ovinas. Madrid: INIA, 1988. $41 \mathrm{p}$.

COSTA, R. G.; LIMA, C. A. C.; MEDEIROS, A. N.; COSTA LIMA, G. F.; MARQUES, C. A. T.; SANTOS, N. M. Características de carcaça de cordeiros Morada Nova alimentados com diferentes níveis do fruto-refugo de melão em substituição ao milho moído na dieta. Revista Brasileira de Zootecnia, Viçosa, MG, v. 40, n. 4, p. 866-871, 2011.

CRANWELL, C. D.; UNRUH, J. A.; BRETHOUR, J. R.; SIMMS, D. D. Influence of steroid implants and concentrate feeding on carcass and longissimus muscle sensory and collagen characteristics of cull beef cows. Journal Animal Science, Manhattan, v. 74, n. 8, p. 17771783, 1996.

CUNHA, E. A.; BUENO, M. S.; SANTOS, L. E.; RODA, D. S.; OTSUK, I. P. Desempenho e características de carcaça de cordeiros suffolk alimentados com diferentes volumosos. Ciência Rural, Santa Maria, v. 31, n. 4, p. 671-676, 2001.

CUNHA, E. A; SANTOS, L. E.; BUENO, M. S.; RODA, D. S.; LEINZ, F. F.; RODRIGUES, C. F. C. Utilização de carneiros de raças de corte para obtenção de cordeiros precoces para abate em plantéis produtores de lã. Revista Brasileira de Zootecnia, Viçosa, MG, v. 29, n. 1, p. 2243252, 2000.

DEVINE, C. E.; CHRYSTALL, B. B.; DAVEY, C. L.; KIRTON, A. H. Effects of nutrition in lambs and subsequent postmortem biochemical changes in muscle. New Zealand of Agricultural Research, Wellington, v. 26, n. 1, p. 53-57, 1983.

FERREIRA, A. C. H.; NEIVA, J. N. M.; RODRIGUEZ, N. M.; SANTANA, G. Z. M.; BORGES, I.; LÔBO, R. N. B. Desempenho produtivo de ovinos alimentados com silagens de capim-elefante contendo resíduos do processamento de frutas. Revista Ciência Agronômica, Fortaleza, v. 40, n. 2, p. 315-322, 2009.

FURUSHO-GARCIA, I. F.; PEREZ, J. R. O.; BONAGURIO, S.; LIMA, A. L.; QUINTÃO, F. A. Estudos dos cortes de carcaça de cordeiros Santa Inês puros e cruzas Santa Inês com Texel, Ile de France e 
Bergamácia. Revista Brasileira de Zootecnia, Viçosa, MG, v. 33, n. 2, p. 453-462, 2004.

GARCIA, C. A. Avaliação do resíduo de panificação "biscoito" na alimentação de ovinos e nas características quantitativas e qualitativas da carcaça.1998. Dissertação (Mestrado em Zootecnia) - Universidade Estadual Paulista, Jaboticabal.

GARCIA, I. F. F.; PEREZ, J. R. O.; OLIVEIRA, M. V. Características de carcaça de cordeiros Texel $\mathrm{x}$ Bergamácia, Texel x Santa Inês e Santa Inês puros, terminados em confinamento, com casca de café como parte da dieta. Revista Brasileira de Zootecnia, Viçosa, MG, v. 29, n. 1, p. 253-260, 2000.

GONÇALVES, L. A. G.; ZAPATA, J. F. F.; RODRIGUES, M. C. P.; BORGES, A. S. Efeitos do sexo e do tempo de maturação sobre a qualidade da carne ovina. Ciência Tecnologia de Alimentos, Campinas, v. 24, n. 3, p. 459467, jul./set. 2004.

GONZAGANETO, S.; BATISTA,A. M.V.; CARVALHO, F. F. R.; MARQUES, C. A. T.; SANTOS, G. R. A. Composição bromatológica, consumo e digestibilidade "in vivo" de dietas com diferentes níveis de feno de catingueira (Caesalpinea bracteosa), fornecidas para ovinos Morada Nova. Revista Brasileira de Zootecnia, Viçosa, MG, v. 30, n. 2, p. 553-562, 2001.

HASHIMOTO, J. H.; OSÓRIO, J. C. S.; OSÓRIO, M. T. M.; BONACINAM, S.; LEHMEN, R. I.; PEDROSO, C. E. S. Qualidade de carcaça, desenvolvimento regional e tecidual de cordeiros terminados em três sistemas. Revista Brasileira de Zootecnia, Viçosa, MG, v. 41, n. 2, p. 438-448, 2012.

HUFF-LONERGAN, E.; LONERGAN, S. M. Mechanisms of water-holding capacity of meat: The role of postmortem biochemical and structural changes. Meat Science, Barking, v. 71, n. 1, p. 194-204, 2005.

LAWLESS, H. T.; HEYMANN, H. Sensory evaluation of food. New York: Chapman \& Hall, 1998. 819 p.

LOUSADA JÚNIOR, J. E.; COSTA, J. M. C.; NEIVA, J. N. M.; RODRIGUEZ N. M. Caracterização físicoquímica de subprodutos obtidos do processamento de frutas tropicais visando seu aproveitamento na alimentação animal. Revista Ciência Agronômica, Fortaleza, v. 37, n. 1, p. 70-76, 2006.

LOUSADA JÚNIOR, J. E.; NEIVA, J. N.; RODRIGUEZ, N. M.; PIMENTEL, J. C. M.; LÔBO, R. N. B. Consumo e digestibilidade aparente de subprodutos do processamento de frutas em ovinos. Revista Brasileira de Zootecnia, Viçosa, MG, v. 34, n. 2, p. 659-669, 2005.
MADRUGA, M. S.; SOUSA, W. H.; ROSALES, M. D.; CUNHA, M. G. G.; RAMOS, J. L. F. Qualidade da carne de cordeiros Santa Inês terminados com diferentes dietas. Revista Brasileira de Zootecnia, Viçosa, MG, v. 34, n. 1, p. 309-315, 2005.

MATIAS, M. F. O.; OLIVEIRA, E. L.; GERTRUDES, E.; MAGALHÂES, M. A. Use of fibres obtained from the cashew (Anacardium ocidentale, L) and guava (Psidium guayava) fruits for enrichment of food products. Brazilian Archives of Biology and Technology, Curitiba, v. 48, p. 143-150, 2005. Special.

NATIONAL RESEARCH COUNCIL - NRC. Nutrient requirements of small ruminants: sheep, goats, cervids, and New World camelids. Washington, D.C.: The National Academies Press, 2007. 362 p.

OLFAZ, M.; OCAK, N.; ERENER, G.; CAM, M. A.; GARIPOGLU, A. V. Growth, carcass and meat characteristics of Karayaka growing rams fed sugar beet pulp, partially substituting for grass hay as forage. Meat Science, Barking, v. 70, n. 1, p. 7-14, 2005.

PINHEIRO, R. S. B.; JORGE, A. M. Medidas biométricas obtidas in vivo e na carcaça de ovelhas de descarte em diferentes estágios fisiológicos. Revista Brasileira Zootecnia, Viçosa, v. 39, n. 2, p. 440-445, 2010.

PINHEIRO, R. S. B.; JORGE, A. M.; MOURÃO, R. C.; POLIZEL NETO, A.; ANDRADE, E. N.; GOMES, H. F. B. Qualidade da carne de cordeiros confinados recebendo diferentes relações de volumoso: concentrado na dieta. Ciência e Tecnologia de Alimentos, Campinas, v. 29, n. 2, p. 407-411, 2009.

RAMOS, E. M.; GOMIDE, L. A. M. Avaliação da qualidade de carnes: fundamento e metodologias. Viçosa: UFV, 2007. 599 p.

RODRIGUES, R. H.; SUSIN, I.; PIRES, A. V.; MENDES, C. Q.; URANO, F. S.; CASTILLO, C. J. C. Polpa cítrica em rações para cordeiros em confinamento: características da carcaça e qualidade da carne. Revista Brasileira Zootecnia, Viçosa, v. 37, n. 10, p. 1869-1875, 2008.

ROTA, E. L.; OSÓRIO, M. T. M.; OSÓRIO, J. C. S.; OLIVEIRA, M. M.; WIEGAND, M. M.; MENDONÇA, G.; ESTEVES, R. M.; GONÇALVES, M. Influência da castração e da idade de abate sobre as características subjetivas e instrumentais da carne de cordeiros Corriedale. Revista Brasileira de Zootecnia, Viçosa, MG, v. 35, n. 6, p. 2397-2405, 2006.

RUSSO, C.; PREZIUSO, G.; CASAROSA, L.; CAMPODONIB, G.; CIAANC, D. Effect of diet energy source on the chemical-physical characteristics of meat and depot fat of lambs carcasses. Small Ruminant Research, Amsterdam, v. 33, n. 1, p. 77-85, 1999. 
SANTOS-SILVA, J.; BESSA, R. J. B.; MENDES, I. A. The effect of supplementation with expanded sunflower seed on carcass and meat quality of lambs raised on pasture. Meat Science, Barking, v. 65, n. 4, p. 1301-1308, dec. 2003.

SAÑUDO, C., SIERRA, I. Calidad de la canal en la espécie ovina. Ovino, One S.A., Barcelona, p. 127-153, sep. 1986.

SCERRA, V.; CAPARRA, P.; FOTI, F.; LANZAB, M.; PRIOLOB, A. Citrus pulp and wheat straw silage as ingredient in lamb diets: effects of growth and carcass and meat quality. Small Ruminant Research, Amsterdam, v. 40, n. 1, p. 51-56, 2001.

SILVA SOBRINHO, A. G.; PURCHAS, R. W.; KADIM, I. T.; YAMAMOTO, S. M. Características de qualidade da carne de ovinos de diferentes genótipos e idades ao abate. Revista Brasileira de Zootecnia, Viçosa, MG, v. 34, n. 3, p. 1070-1078, 2005.

SILVA, L. M.; OLIVEIRA, C. H. A.; RODRIGUES, F. V.; RODRIGUES, M. R. C.; BESERRA, F. J.; SILVA, A. M.; LEMOS, J. C.; FERNANDES, A. A. O.; RONDINA, D. Desempenho e características da carcaça de cordeiros alimentados com bagaço de caju. Archivos de Zootecnia, Córdoba, v. 60, n. 231, p. 777-786, 2011.

SILVA, N. V.; COSTA R, G.; MEDEIROS, A. N.; AZEVEDO, P. S.; CARVALHO, F. F. R.; MEDEIROS, G. R.; MADRUGA, M. S. Efeito do feno de flor-de-seda sobre a carcaça e constituintes corporais de cordeiros Morada Nova. Archivos de Zootecnia, Córdoba, v. 61, n. 233, p. 63-70, 2012.

SIQUEIRA, E. R.; SIMÕES, C. D.; FERNANDES, S. Efeito do sexo e do peso ao abate sobre a produção de carne de cordeiro. Morfometria da carcaça, pesos dos cortes, composição tecidual e componentes nãoconstituintes da carcaça. Revista Brasileira de Zootecnia, Viçosa, MG, v. 30, n. 4, p. 1299-1307, 2001.
SOARES, B. C.; SOUZA, K. D. S.; LOURENÇO JUNIOR, J. B.; MACIEL E SILVA, A. G.; ÁVILA, S. C.; KUSS, F.; ANDRADE, S. J. T.; RAIOL, L. C. B.; COLODO, J. C. N. Desempenho e características de carcaças de cordeiros suplementados com diferentes níveis de resíduo de biodiesel. Arquivo Brasileiro de Medicina Veterinária e Zootecnia, Pará, v. 64, n. 6, p. 1747-1754, 2012.

SOUSA, W. H.; BRITO, E. A.; MEDEIROS, A. N.; CARTAXO, F. Q.; CEZAR, M. F.; CUNHA, M. D. G. G. Características morfométricas e de carcaça de cabritos e cordeiros terminados em confinamento. Revista Brasileira de Zootecnia, Viçosa, MG, v. 38, n. 7, p. 13401346, 2009.

STATISTICAL ANALYSIS SYSTEM - SAS. User's guide. Cary: SAS Institute, 2002. 525 p.

XENOFONTE, A. R. B.; CARVALHO, F. F. R.; BATISTA, A. M. V.; MEDEIROS, G. R. Características de carcaça de ovinos em crescimento alimentados com rações contendo farelo de babaçu. Revista Brasileira de Zootecnia, Viçosa, MG, v. 38, n. 2, p. 392-398, 2009.

ZEOLA, N. M. B. L.; SILVA SOBRINHO, A. G.; GONZAGA NETO, S. Efeito da relação volumoso: concentrado sobre a qualidade da carne de ovinos Morada Nova. In: CONGRESSO BRASILEIRO DE CIÊNCIA E TECNOLOGIA DE CARNES, 1., 2001, São Pedro. Anais... São Pedro: Editora Universitária, 2001. p. 104105.

ZEOLA, N. M. B. L.; SOUZA, P. A.; SOUZA, H. B. A.; SILVA SOBRINHO, A. G. Parâmetros qualitativos da carne ovina: um enfoque à maturação e marinação. Revista Portuguesa de Ciências Veterinárias, Lisboa, v. 102, n. 563-564, p. 215-224, 2007. 
